DEMONSTRATIO MATHEMATICA

Vol. XXXVIII No $3 \quad 2005$

Mohamed Akkouchi, M. H. Lalaoui Rhali

\title{
STUDY OF SOME FUNCTIONAL EQUATIONS RELATED TO SOME ALGEBRAS OF MATRICES
}

\begin{abstract}
Let $\mathbb{K}$ be the real or complex field. Let $1 \leq n \leq p$ be two positive integers. We denote $\mathcal{M}_{p}(\mathbb{K})$ the usual algebra of $p \times p$-matrices. Let $\mathcal{A}_{n}$ be an $n$-dimensional subalgebra of $\mathcal{M}_{p}(\mathbb{K})$. Then there exists an injective linear mapping $A: \mathbb{K}^{n} \rightarrow \mathcal{M}_{p}(\mathbb{K})$ such that $A\left(\mathbb{K}^{n}\right)=\mathcal{A}_{n}$. Therefore $\mathbb{K}^{n}$ may be equipped with a product denoted $\star$ such that $\left(\mathbb{K}^{n},+, \star\right)$ is an associative algebra. The aim of this paper is to investigate the general solutions of the functional equation: $f(x \star y)=f(x) f(y)$, and its Pexider form $f(x \star y)=$ $g(x) h(y)$, for all $x, y$ in $\mathbb{K}^{n}$, where $f, g, h: \mathbb{K}^{n} \rightarrow \mathbb{K}$ are unknown functions. This work is inspired by the paper [2].
\end{abstract}

\section{Introduction, notations and examples}

1.1. Throughout this paper, $\mathbb{K}$ will be the real or complex field. Let $n, p$ be two positive integers such that $1 \leq n \leq p$. Let $A: \mathbb{K}^{n} \rightarrow \mathcal{M}_{p}(\mathbb{K})$ be a mapping, where $\mathcal{M}_{p}(\mathbb{K})$ is the usual algebra of $p \times p$-matrices, such that the following conditions are satisfied:

(C 1$)$ The mapping $A$ is linear and injective,

(C 2) $A\left(\mathbb{K}^{n}\right) A\left(\mathbb{K}^{n}\right) \subset A\left(\mathbb{K}^{n}\right)$, where $A\left(\mathbb{K}^{n}\right)$ is the range of $A$.

Then conditions (C 1$)$ and (C 2) imply that for all $x, y$ in $\mathbb{K}^{n}$ there exists an unique element $z \in \mathbb{K}^{n}$ such that $A(z)=A(x) A(y)$. We denote $z=x \star y$. It is obvious that $\left(\mathbb{K}^{n},+, \star\right)$ is an associative algebra. By (C 1$)$ and (C 2), this algebra is isomorphic to a subalgebra of $\mathcal{M}_{p}(\mathbb{K})$ and that $A$ is an algebraisomorphism.

The aim of this paper is to find the general solution of the functional equation

$$
f(x \star y)=f(x) f(y), \quad \forall x, y \in \mathbb{K}^{n},
$$

1991 Mathematics Subject Classification: Primary 39B22.

Key words and phrases: functional equation, multiplicative function, algebra of matrices. 
and its Pexider form

$$
f(x \star y)=g(x) h(y), \quad \forall x, y \in \mathbb{K}^{n},
$$

where $f, g, h: \mathbb{K}^{n} \rightarrow \mathbb{K}$ are unknown functions under the condition:

(C 3) There exists a set $\left\{v_{1}, \ldots, v_{n}\right\}$ of linearly independent vectors in the linear space $\mathbb{K}^{p}$ and a set $\left\{f_{1}, \ldots, f_{n}\right\}$ of linearly independent linear functionals on $\mathbb{K}^{n}$ such that

$$
A(x)\left(v_{i}\right)=f_{i}(x) v_{i}, \quad \forall i=1,2, \ldots n, \quad \text { and } \quad x \in \mathbb{K}^{n} .
$$

1.2. Remarks and notations: a) We remark that condition (C 3 ) requires $n \leq p$.

b) In all this paper, for all integers $q \geq 1$, we identify every endomorphism $T$ on $\mathbb{K}^{q}$ with its matrix $\mathbf{T}$ relative to the natural basis of $\mathbb{K}^{q}$. This explains the meaning of the condition (C 3 ) above. Also, we identify any vector $u \in \mathbb{K}^{q}$ with its matrix of coordinates $\mathbf{u}=\left(u_{1}, \ldots, u_{q}\right)$, which is a matrix of type $1 \times q$. With the above notations, we write $\mathbf{u}^{t} \mathbf{T}$ or $u^{t} T$ to designate the coordinate matrix of the vector $T(u)$, the image of $u$ under $T$, where ${ }^{t} T$ designates the transpose of the matrix (operator) $T$.

c) We point out that the paper [2] deals with particular cases of equations (1.1) and (1.2).

Before discussing the validity of the conditions (C 1), (C 2) and (C 3) given above, we provide some examples.

\subsection{Examples}

1.3.1. $n=2$ and $p=3$. Consider the set $\mathcal{A}_{2}$ of matrices $A\left(x_{1}, x_{2}\right) \in \mathcal{M}_{3}(\mathbb{K})$ given by

$$
A\left(x_{1}, x_{2}\right):=\left(\begin{array}{ccc}
x_{1} & x_{2} & 0 \\
x_{2} & x_{1} & 0 \\
0 & 0 & x_{1}-x_{2}
\end{array}\right)
$$

for all $\left(x_{1}, x_{2}\right) \in \mathbb{K}^{2}$. Then $\mathcal{A}_{2}$ is an unitary commutative subalgebra of $\mathcal{M}_{3}(\mathbb{K})$ of dimension 2 . The mapping $A$ defined above is linear and bijective from $\mathbb{K}^{2}$ onto $\mathcal{A}_{2}$ and the corresponding algebra structure on $\mathbb{K}^{2}$ is

$$
x \star y:=\left(x_{1} y_{1}+x_{2} y_{2}, x_{1} y_{2}+x_{2} y_{1}\right),
$$

for all $x=\left(x_{1}, x_{2}\right), y=\left(y_{1}, y_{2}\right) \in \mathbb{K}^{2}$. The condition (C 3) is satisfied. Indeed, the vectors $v_{1}:=(1,1,0)$ and $v_{2}:=(1,-1,0)$ are linearly independent in $\mathbb{K}^{3}$ and we have

$A(x)\left(v_{1}\right)=\left(x_{1}+x_{2}\right) v_{1}:=f_{1}(x) v_{1}$, and $A(x)\left(v_{1}\right)=\left(x_{1}-x_{2}\right) v_{2}:=f_{2}(x) v_{2}$.

It is clear that the set $\left\{f_{1}, f_{2}\right\}$ of linear functionals (defined above) is a basis of the dual space of the linear space $\mathbb{K}^{2}$. 
1.3.2. $n=2$ and $p=4$. Consider the set $\mathcal{A}_{2}$ of matrices $A\left(x_{1}, x_{2}\right) \in \mathcal{M}_{4}(\mathbb{K})$ given by

$$
A\left(x_{1}, x_{2}\right):=\left(\begin{array}{llll}
x_{1} & x_{2} & x_{1} & x_{2} \\
x_{2} & x_{1} & x_{2} & x_{1} \\
x_{1} & x_{2} & x_{1} & x_{2} \\
x_{2} & x_{1} & x_{2} & x_{1}
\end{array}\right)
$$

for all $\left(x_{1}, x_{2}\right) \in \mathbb{K}^{2}$. Then $\mathcal{A}_{2}$ is an unitary commutative subalgebra of $\mathcal{M}_{4}(\mathbb{K})$ of dimension 2. The mapping $A$ defined above is linear and bijective from $\mathbb{K}^{2}$ onto $\mathcal{A}_{2}$ and the corresponding algebra structure on $\mathbb{K}^{2}$ is

$$
x \star y:=\left(x_{1} y_{1}+x_{2} y_{2}, x_{1} y_{2}+x_{2} y_{1}\right),
$$

for all $x=\left(x_{1}, x_{2}\right), y=\left(y_{1}, y_{2}\right) \in \mathbb{K}^{2}$. The condition (C 3$)$ is satisfied. Indeed, the vectors $v_{1}:=(1,1,1,1)$ and $v_{2}:=(1,-1,1,-1)$ are linearly independent in $\mathbb{K}^{4}$ and we have $A(x)\left(v_{1}\right)=2\left(x_{1}+x_{2}\right) v_{1}:=f_{1}(x) v_{1}$, and $A(x)\left(v_{2}\right)=2\left(x_{1}-x_{2}\right) v_{2}:=f_{2}(x) v_{2}$. It is clear that the set $\left\{f_{1}, f_{2}\right\}$ of linear functionals (defined above) is a basis of the dual space of the linear space $\mathbb{K}^{2}$.

1.3.3. $n=2$ and $p \geq 2$. Consider the set $\mathcal{A}_{2}$ of matrices $A\left(x_{1}, x_{2}\right) \in \mathcal{M}_{p}(\mathbb{K})$ given by

$$
A\left(x_{1}, x_{2}\right):=\left(\begin{array}{ccccc}
x_{1} & x_{2} & x_{2} & \ldots & x_{2} \\
x_{2} & x_{1} & x_{2} & \ldots & x_{2} \\
\vdots & \ddots & \ddots & \ddots & \vdots \\
x_{2} & \ldots & x_{2} & x_{1} & x_{2} \\
x_{2} & \ldots & x_{2} & x_{2} & x_{1}
\end{array}\right)
$$

for all $\left(x_{1}, x_{2}\right) \in \mathbb{K}^{2}$. Then $\mathcal{A}_{2}$ is an unitary commutative subalgebra of $\mathcal{M}_{p}(\mathbb{K})$ of dimension 2. The mapping $A$ defined above is linear and bijective from $\mathbb{K}^{2}$ onto $\mathcal{A}_{2}$ and the corresponding algebra structure on $\mathbb{K}^{2}$ is

$$
x \star y:=\left(x_{1} y_{1}+(p-1) x_{2} y_{2}, x_{1} y_{2}+x_{2} y_{1}+(p-2) x_{2} y_{2}\right),
$$

for all $x=\left(x_{1}, x_{2}\right), y=\left(y_{1}, y_{2}\right) \in \mathbb{K}^{2}$. The condition (C 3$)$ is satisfied, by taking the vectors $v_{1}:=(1,1,1, \ldots, 1)$ and $v_{2}:=(1,-1,0, \ldots, 0)$ in $\mathbb{K}^{p}$. These vectors are linearly independent in $\mathbb{K}^{p}$ and we have

$$
A(x)\left(v_{1}\right)=\left(x_{1}+(p-1) x_{2}\right) v_{1}:=f_{1}(x) v_{1},
$$

and

$$
A(x)\left(v_{2}\right)=\left(x_{1}-x_{2}\right) v_{2}:=f_{2}(x) v_{2} .
$$

It is clear that the set $\left\{f_{1}, f_{2}\right\}$ of linear functionals (defined above) is a basis of the dual space of the linear space $\mathbb{K}^{2}$. 
Next we quote two examples showing that the matrix $A(x)$ may not be symmetric.

1.3.4. $n=2$ and $p=3$. Consider the set $\mathcal{A}_{2}$ of matrices $A\left(x_{1}, x_{2}\right) \in \mathcal{M}_{3}(\mathbb{K})$ given by

$$
A\left(x_{1}, x_{2}\right):=\left(\begin{array}{ccc}
x_{1}+3 x_{2} & -x_{2} & x_{2} \\
0 & x_{1}+2 x_{2} & 0 \\
x_{2} & -x_{2} & x_{1}+3 x_{2}
\end{array}\right)
$$

for all $\left(x_{1}, x_{2}\right) \in \mathbb{K}^{2}$. Then $\mathcal{A}_{2}$ is an unitary commutative subalgebra of $\mathcal{M}_{3}(\mathbb{K})$ of dimension 2. The mapping $A$ defined above is linear and bijective from $\mathbb{K}^{2}$ onto $\mathcal{A}_{2}$ and the corresponding algebra structure on $\mathbb{K}^{2}$ is

$$
x \star y:=\left(x_{1} y_{1}-8 x_{2} y_{2}, x_{1} y_{2}+x_{2} y_{1}+6 x_{2} y_{2}\right),
$$

for all $x=\left(x_{1}, x_{2}\right), y=\left(y_{1}, y_{2}\right) \in \mathbb{K}^{2}$. The condition (C 3$)$ is satisfied. Indeed, the vectors $v_{1}:=(1,0,-1)$ and $v_{2}:=(1,0,1)$ are linearly independent in $\mathbb{K}^{3}$ and we have

$$
A(x)\left(v_{1}\right)=\left(x_{1}+2 x_{2}\right) v_{1}:=f_{1}(x) v_{1}
$$

and

$$
A(x)\left(v_{1}\right)=\left(x_{1}+4 x_{2}\right) v_{2}:=f_{2}(x) v_{2} .
$$

It is obvious that the linear functionals $f_{1}$ and $f_{2}$ (defined above) are linearly independent.

1.3.5. $n=2$ and $p=4$. Consider the set $\mathcal{A}_{2}$ of matrices $A\left(x_{1}, x_{2}\right) \in \mathcal{M}_{4}(\mathbb{K})$ given by

$$
A\left(x_{1}, x_{2}\right):=\left(\begin{array}{cccc}
x_{1}+x_{2} & -2 x_{2} & 4 x_{2} & -6 x_{2} \\
2 x_{2} & x_{1}-x_{2} & 2 x_{2} & 0 \\
0 & 6 x_{2} & x_{1}-3 x_{2} & 6 x_{2} \\
-2 x_{2} & 4 x_{2} & -2 x_{2} & x_{1}+3 x_{2}
\end{array}\right)
$$

for all $\left(x_{1}, x_{2}\right) \in \mathbb{K}^{2}$. Then $\mathcal{A}_{2}$ is an unitary commutative subalgebra of $\mathcal{M}_{4}(\mathbb{K})$ of dimension 2 . The mapping $A$ defined above is linear and bijective from $\mathbb{K}^{2}$ onto $\mathcal{A}_{2}$ and the corresponding algebra structure on $\mathbb{K}^{2}$ is

$$
x \star y:=\left(x_{1} y_{1}+9 x_{2} y_{2}, x_{1} y_{2}+x_{2} y_{1}\right),
$$

for all $x=\left(x_{1}, x_{2}\right), y=\left(y_{1}, y_{2}\right) \in \mathbb{K}^{2}$. The condition (C 3$)$ is satisfied. Indeed, the vectors $v_{1}:=(1,0,-1,0)$ and $v_{2}:=(-1,0,1,1)$ are linearly independent in $\mathbb{K}^{4}$ and we have

$$
A(x)\left(v_{1}\right)=\left(x_{1}-3 x_{2}\right) v_{1}:=f_{1}(x) v_{1},
$$

and

$$
A(x)\left(v_{2}\right)=\left(x_{1}+3 x_{2}\right) v_{2}:=f_{2}(x) v_{2} .
$$

It is obvious that the linear functionals $f_{1}$ and $f_{2}$ are linearly independent. 
Other examples are studied at the end of this paper. Moreover, in Section 2, the existence of mappings $A$ satisfying conditions (C 1), (C 2) and (C 3) for any $n$ and $p$ with $1 \leq n \leq p$ will be shown.

1.4. Organization of the paper. This paper is organized as follows: In Section 2, the problem of existence of mappings $A$ satisfying conditions (C 1), (C 2) and (C 3) for any integers $n$ and $p$ with $1 \leq n \leq p$ will be solved (see Theorem 2.1). In Section 3, we determine the general solution of equation (1.1) (see Theorem 3.1). In Section 4, when in addition the algebra $\left(\mathbb{K}^{n},+, \star\right)$ has a unit, we solve equation (1.2) (see Theorem 4.1). In the last section, we give illustrative examples. These examples show, in particular, how the results obtained by J. K. Chung and P. K. Sahoo in [2] can be derived from the present paper.

We point out that the methods used here to solve equations (1.1) and (1.2) are purely algebraic and do not require any regularity assumptions on the unknown functions.

\section{An existence result}

Our first result is the following

THEOREM 2.1. Let $p \geq 1$ be an integer. Then, for each integer $1 \leq n \leq p$, there exists a mapping $A: \mathbb{K}^{n} \rightarrow \mathcal{M}_{p}(\mathbb{K})$ satisfying the following conditions: (C 1) The mapping $A$ is linear and injective,

(C 2) $A\left(\mathbb{K}^{n}\right) A\left(\mathbb{K}^{n}\right) \subset A\left(\mathbb{K}^{n}\right)$, and

(C 3) there exists a set $\left\{v_{1}, \ldots, v_{n}\right\}$ of linearly independent vectors in $\mathbb{K}^{p}$ and a set $\left\{f_{1}, \ldots, f_{n}\right\}$ of linearly independent linear functionals on $\mathbb{K}^{n}$ such that

$$
A(x)\left(v_{i}\right)=f_{i}(x) v_{i}, \quad \forall i=1,2, \ldots n, \quad \text { and } \quad x \in \mathbb{K}^{n} .
$$

P roof. Let $G \in \mathcal{M}_{p}(\mathbb{K})$ be a diagonalizable matrix having exactly $n$ distinct eigenvalues $\lambda_{1}, \lambda_{2}, \ldots, \lambda_{n}$. The minimal polynomial of $G$ is given by $Q(X)=$ $\prod_{i=1}^{n}\left(X-\lambda_{i}\right)$. Since $Q(G)=0$ then the set of matrices $\left\{I_{p}, G, \ldots, G^{n-1}\right\}$ is linearly independent in $\mathcal{M}_{p}(\mathbb{K})$ where, $I_{p}$ is the unit matrix. Let $\mathcal{A}_{n}(G)$ be the linear span of the set $\left\{I_{p}, G, \ldots, G^{n-1}\right\}$. Then $\mathcal{A}_{n}(G)$ is a commutative unitary subalgebra of $\mathcal{M}_{p}(\mathbb{K})$. We consider the mapping $A$ from $\mathbb{K}^{n}$ to $\mathcal{M}_{p}(\mathbb{K})$ given by:

$$
A\left(x_{0}, x_{1}, \ldots, x_{n-1}\right):=\sum_{i=0}^{n-1} x_{i} G^{i}
$$

It is clear that conditions (C 1) and (C 2) are satisfied. To show the condition (C 3$)$, we consider a basis in $\mathbb{K}^{p}\left\{v_{1}, v_{2}, \ldots, v_{n}, \ldots, v_{p}\right\}$ such that

$$
G\left(v_{i}\right)=\lambda_{i} v_{i}, \quad \forall i=1, \ldots, n \text {. }
$$


For all $x=\left(x_{0}, x_{1}, \ldots, x_{n-1}\right) \in \mathbb{K}^{n}$, we set

$$
f_{i}(x):=\sum_{j=0}^{n-1} \lambda_{i}{ }^{j} x_{j}, \quad \forall i=1, \ldots, n .
$$

Then $f_{1}, \ldots, f_{n}$ are linear functionals defined on $\mathbb{K}^{n}$, and it is easy to see that they are linearly independent.

Now, for each $x=\left(x_{0}, x_{1}, \ldots, x_{n-1}\right) \in \mathbb{K}^{n}$, we have

$$
A(x)\left(v_{i}\right)=\sum_{j=0}^{n-1} x_{j} G^{j}\left(v_{i}\right)=\left[\sum_{j=0}^{n-1} \lambda_{i}^{j} x_{j}\right] v_{i},=f_{i}(x) v_{i} \quad \forall i=1, \ldots, n .
$$

Therefore, the condition (C 3 ) is also satisfied. This completes the proof.

\section{Solutions of equation (1.1)}

Let $n, p$ be two natural integers such that $1 \leq n \leq p$. Let $A: \mathbb{K}^{n} \rightarrow$ $\mathcal{M}_{p}(\mathbb{K})$ satisfy the conditions (C 1), (C 2) and (C 3 ).

We recall that a mapping $M: \mathbb{K} \longrightarrow \mathbb{K}$ is said to be multiplicative if $M(x y)=M(x) M(y)$ for all $x, y \in \mathbb{K}$.

The following theorem is the main result of this paper.

THEOREM 3.1. A mapping $f: \mathbb{K}^{n} \longrightarrow \mathbb{K}$ is a solution of (1.1), if and only if there exist $n$ multiplicative mappings $M_{1}, \ldots, M_{n}: \mathbb{K} \longrightarrow \mathbb{K}$ such that

$$
f(x)=M_{1}\left(f_{1}(x)\right) M_{2}\left(f_{2}(x)\right) \ldots M_{n}\left(f_{n}(x)\right),
$$

for all $x \in \mathbb{K}^{n}$ where $f_{1}, \ldots, f_{n}: \mathbb{K}^{n} \longrightarrow \mathbb{K}$ are the linear functionals from (C 3).

Proof. Let $f: \mathbb{K}^{n} \longrightarrow \mathbb{K}$ be a solution of the functionnal equation (1.1). For each $x=\left(x_{1}, \ldots, x_{n}\right) \in \mathbb{K}^{n}$, we set $X=\left(X_{1}, \ldots, X_{n}\right)$, where

$$
X_{i}=f_{i}\left(x_{1}, \ldots, x_{n}\right), \quad \forall i=1,2, \ldots, n .
$$

The equalities in (3.2) above may be written in the form

$$
X=P(x),
$$

where $P$, according to our assumptions, is a linear invertible operator. Let $P^{-1}$ be the inverse of $P$ and let us set

$$
F(x)=f\left(P^{-1}(x)\right), \quad \forall x \in \mathbb{K}^{n} .
$$

From (3.4) we get

$$
f(x)=F(P(x)), \quad \forall x \in \mathbb{K}^{n} .
$$

We want to find a functional equation satisfied by the mapping $F$. To this end, let $X, Y \in \mathbb{K}^{n}$. Then we can find in a unique manner $x, y \in \mathbb{K}^{n}$, such that

$$
X=P(x) \quad \text { and } \quad Y=P(y) .
$$


Denote $z=x \star y$ and $Z=P(z)=\left(Z_{1}, \ldots, Z_{n}\right)$, where

$$
Z_{i}=f_{i}(x \star y), \quad \forall i=1,2, \ldots, n .
$$

We observe that for each $i=1, \ldots, n$ we have

$$
f_{i}(x \star y)=f_{i}(x) f_{i}(y), \quad \forall x, y \in \mathbb{K}^{n} .
$$

Indeed, for all $x, y \in \mathbb{K}^{n}$ and for each $i=1, \ldots, n$, we have

$$
A(x \star y)\left(v_{i}\right)=f_{i}(x \star y) v_{i}
$$

and that

$$
A(x \star y)\left(v_{i}\right)=A(x) A(y)\left(v_{i}\right)=f_{i}(y) A(x)\left(v_{i}\right)=f_{i}(y) f_{i}(x) v_{i} .
$$

We conclude that $Z=\left(X_{1} Y_{1}, \ldots, X_{n} Y_{n}\right)$. Therefore, we obtain

$$
\begin{aligned}
F\left(X_{1} Y_{1}, \ldots, X_{n} Y_{n}\right) & =F(P(z))=f\left(P^{-1}(P(z))\right)=f(z) \\
& =f(x \star y)=f(x) f(y) \\
& =F(P(x)) F(P(y))=F(X) F(Y) .
\end{aligned}
$$

Consequently $F\left(X_{1} Y_{1}, \ldots, X_{n} Y_{n}\right)=F\left(X_{1}, \ldots, X_{n}\right) F\left(Y_{1}, \ldots, Y_{n}\right)$, for all $X=\left(X_{1}, \ldots, X_{n}\right)$ and $Y=\left(Y_{1}, \ldots, Y_{n}\right)$ in $\mathbb{K}^{n}$. Then all the functions defined from $\mathbb{K}$ to $\mathbb{K}$ by:

$$
\begin{aligned}
& M_{1}: X_{1} \longrightarrow F\left(X_{1}, 1, \ldots, 1\right), \\
& M_{i}: X_{i} \longrightarrow F\left(1, \ldots, 1, X_{i}, 1, \ldots, 1\right), \quad 1<i<n, \\
& M_{n}: X_{n} \longrightarrow F\left(1, \ldots, X_{n}\right),
\end{aligned}
$$

are multiplicative. Moreover, for each $X=\left(X_{1}, \ldots, X_{n}\right)$ in $\mathbb{K}^{n}$, we have

$$
\begin{aligned}
F(X) & =F\left(X_{1}, 1, \ldots 1\right) F\left(1, X_{2}, 1 . ., 1\right) \ldots F\left(1, \ldots 1, X_{n}\right) \\
& =\prod_{i=1}^{n} M_{i}\left(X_{i}\right) .
\end{aligned}
$$

Therefore, from (3.9), for all $x \in \mathbb{K}^{n}$, we get

$$
f(x)=F(P(x))=F\left(f_{1}(x), \ldots, f_{n}(x)\right)=\prod_{i=1}^{n} M_{i}\left(f_{i}(x)\right) .
$$

So we have the desired identity (3.1) for $f$. Conversely, if $f$ is given by (3.1), where $M_{i}: \mathbb{K} \longrightarrow \mathbb{K}$ are multiplicative mappings for $i=1, \ldots, n$, then by using (3.8), it is easy to see that $f$ satisfies equation (1.1). This completes the proof.

\section{Solutions of equation (1.2)}

In this section, all conditions of Section 3 are assumed. To find the solutions of the equation (1.2), we shall need the following extra assumption: 
(U) There exists an element $e \in \mathbb{K}^{n}$ such that

$$
e \star x=x \star e=x, \quad \forall x \in \mathbb{K}^{n} .
$$

This means that the associative algebera $\left(\mathbb{K}^{n},+, \star\right)$ has a unit. For the associated mapping $A$, this condition is equivalent to $A(e) A(x)=A(x) A(e)=$ $A(x)$ for all $x \in \mathbb{K}^{n}$.

Under these assumptions we find the solutions of equation (1.2). They are described in the following theorem.

ThEOREM 4.1. The functions $f, g, h: \mathbb{K}^{n} \rightarrow \mathbb{K}$ satisfy the functional equation (1.2), if and only if

$$
f=0, g=0 \text { and } h \text { is arbitrary }
$$

or

$$
f=0, h=0, \text { and } g \text { is arbitrary }
$$

or

$$
\begin{aligned}
& f(x)=\alpha \beta \prod_{i=1}^{n} M_{i}\left(f_{i}(x)\right), \\
& g(x)=\beta \prod_{i=1}^{n} M_{i}\left(f_{i}(x)\right), \\
& h(x)=\alpha \prod_{i=1}^{n} M_{i}\left(f_{i}(x)\right),
\end{aligned}
$$

for all $x \in \mathbb{K}$, where $M_{1}, \ldots, M_{n}: \mathbb{K} \rightarrow \mathbb{K}$ are multiplicative mappings and $\alpha, \beta$ are nonzero arbitrary constants in $\mathbb{K}$.

Proof. By taking $y=e$ in equation (1.2), we get

$$
f(x)=g(x) h(e)=\alpha g(x) \quad \forall x \in \mathbb{K}^{n},
$$

where $\alpha=h(e)$. Similarly, by taking $x=e$ in (1.2), we get

$$
f(y)=g(e) h(y)=\beta h(y), \quad \forall y \in \mathbb{K}^{n}
$$

where $\beta=g(e)$.

If $\alpha=0$ and $\beta \neq 0$, then $f=0, h=0$ and $g$ is arbitrary.

If $\beta=0$ and $\alpha \neq 0$, then $f=0, g=0$ and $h$ is arbitrary.

If $\alpha \neq 0$ and $\beta \neq 0$, then from equation (1.2), we see that the function $\frac{f}{\alpha \beta}$ satisfies equation (1.1), so by Theorem 2.1 , we deduce that $\frac{f}{\alpha \beta}=$ $M_{1}\left(f_{1}\right) \ldots M_{n}\left(f_{n}\right)$, where $M_{1}, \ldots, M_{n}: \mathbb{K} \rightarrow \mathbb{K}$ are multiplicative mappings. Thus we obtain the identities (4.2), and this completes the proof.

\section{Examples and applications}

In this section we give some examples and applications of our results obtained in the previous sections.

5.1. Example 1. Consider the following functional equations:

$$
f(u x+v y, x v+y u)=f(x, y) f(u, v),
$$


and the associated Pexider equation:

$E_{p}(2 ; 2) \quad f(u x+v y, x v+y u)=g(x, y) h(u, v)$,

where $f, g, h: \mathbb{R}^{2} \longrightarrow \mathbb{R}$ are unknown functions. These equation were studied and solved in [2]. Our results may be applied here. Indeed, let us consider the mapping $A: \mathbb{R}^{2} \longrightarrow \mathcal{M}_{2}(\mathbb{R})$ defined by

$$
A(x, y)=\left(\begin{array}{ll}
x & y \\
y & x
\end{array}\right), \quad(x, y) \in \mathbb{R}^{2} .
$$

Then $A$ is linear and injective, and its range $A\left(\mathbb{R}^{2}\right)$ is a subalgebra with unit of $\mathcal{M}_{2}(\mathbb{R})$. Thus, conditions (C 1), (C2) and (U) are satisfied. It is easy to see that $(\mathrm{C} 3)$ is also satisfied, by taking the vectors

$$
v_{1}=(1,1), \quad v_{2}=(1,-1),
$$

and the linear functionals

$$
f_{1}(x, y)=x+y, \quad f_{2}(x, y)=x-y .
$$

It follows from Theorem 2.1, that the solutions of equation $E(2 ; 2)$ are given by

$$
f(x, y)=M_{1}(x+y) M_{2}(x-y),
$$

where $M_{1}$ and $M_{2}$ are multiplicative mappings from $\mathbb{R}$ to $\mathbb{R}$. This result is Theorem 1 of [2]. From Theorem 4.1 we deduce that the solutions of equation $E_{p}(2 ; 2)$ are given by

$$
f=0, g=0, \text { and } h \text { is arbitrary }
$$

or

$$
f=0, h=0, \text { and } g \text { is arbitrary }
$$

or

$$
\begin{aligned}
& f(x, y)=\alpha \beta M_{1}(x+y) M_{2}(x-y), \\
& g(x, y)=\beta M_{1}(x+y) M_{2}\left(x_{6}-y\right), \\
& h(x, y)=\alpha M_{1}(x+y) M_{2}(x-y),
\end{aligned}
$$

where $M_{1}, M_{2}: \mathbb{R} \rightarrow \mathbb{R}$ are multiplicative mappings and $\alpha, \beta$ are arbitrary nonzero real constants. So we obtain Theorem 2 of the paper [2].

5.2. Example 2. Let $\mathbb{K}$ be the field of real or complex numbers and let $\lambda \in \mathbb{K} \backslash\{0\}$ be a fixed scalar. Consider the following functional equations:

$E(3 ; \lambda) f(u x+(\lambda-1) v y, x v+y u+(\lambda-2) y v, z w)=f(x, y, z) f(u, v, w)$, and the associated Pexider equation:

$E_{p}(3 ; \lambda) f(u x+(\lambda-1) v y, x v+y u+(\lambda-2) y v, z w)=g(x, y, z) h(u, v, w)$, 
where $f, g, h: \mathbb{K}^{3} \longrightarrow \mathbb{K}$ are unknown functions. We may apply our results to solve these equations. Indeed, let us consider the mapping $A_{\lambda}: \mathbb{K}^{3} \longrightarrow$ $\mathcal{M}_{3}(\mathbb{K})$ defined by

$$
A_{\lambda}(x, y, z)=\left(\begin{array}{ccc}
x & 0 & y \\
0 & z & 0 \\
(\lambda-1) y & 0 & x+(\lambda-2) y
\end{array}\right), \quad(x, y, z) \in \mathbb{K}^{3} .
$$

The mapping $A_{\lambda}$ is linear and injective, and for all $(x, y, z),(u, v, w)$ in $\mathbb{K}^{3}$ we have

$$
A_{\lambda}(x, y, z) A_{\lambda}(u, v, w)=A_{\lambda}(X, Y, Z)
$$

where

$$
\begin{aligned}
& X=u x+(\lambda-1) v y, \\
& Y=x v+y u+(\lambda-2) y v, \\
& Z=z w .
\end{aligned}
$$

Thus the condition (U) holds with $e=(1,0,1)$.

It is easy to see that condition (C 3 ) is satisfied, by taking the vectors

$$
v_{1}=(1,0, \lambda-1), \quad v_{2}=(1,0,-1), \quad v_{3}=(0,1,0),
$$

and the linear functionals

$$
f_{1}(x, y, z)=x+(\lambda-1) y, \quad f_{2}(x, y, z)=x-y, \quad f_{3}(x, y, z)=z .
$$

It follows from Theorem 4.1 that the solutions of equation $E(3 ; \lambda)$ are given by

$$
f(x, y, z)=M_{1}(x+(\lambda-1) y) M_{2}(x-y) M_{3}(z),
$$

where $M_{1}, M_{2}$ and $M_{3}$ are multiplicative mappings from $\mathbb{K}$ to $\mathbb{K}$.

From Theorem 3.1 we deduce that the solutions of equation $E_{p}(3 ; \lambda)$ are given by

$$
f=0, g=0, \text { and } h \text { is arbitrary }
$$

or

$$
f=0, h=0, \text { and } g \text { is arbitrary }
$$

or

$$
\begin{aligned}
& f(x, y, z)=\alpha \beta M_{1}(x+(\lambda-1) y) M_{2}(x-y) M_{3}(z), \\
& g(x, y, z)=\beta M_{1}(x+(\lambda-1) y) M_{2}(x-y) M_{3}(z), \\
& h(x, y, z)=\alpha M_{1}(x+(\lambda-1) y) M_{2}(x-y) M_{3}(z),
\end{aligned}
$$

where $M_{1}, M_{2}, M_{3}: \mathbb{K} \rightarrow \mathbb{K}$ are multiplicative mappings and $\alpha, \beta$ are arbitrary nonzero constants.

In the case where $\lambda=2$ and $\mathbb{K}=\mathbb{R}$, we obtain Theorem 3 and Theorem 4 of [2]. 
5.3. Example 3. Let $\mathbb{K}=\mathbb{C}$ be the field of complex numbers and consider the following functional equations:

$E(3) f(u x+w y+v z, v x+u y+w z, w x+v y+u z)=f(x, y, z) f(u, v, w)$, and the associated Pexider equation:

$E_{p}(3) f(u x+w y+v z, v x+u y+w z, w x+v y+u z)=g(x, y, z) h(u, v, w)$, where $f, g, h: \mathbb{C}^{3} \longrightarrow \mathbb{C}$ are unknown functions. We may apply our results to solve these equations. Indeed, let us consider the mapping $A: \mathbb{C}^{3} \longrightarrow \mathcal{M}_{3}(\mathbb{C})$ defined by

$$
A(x, y, z)=\left(\begin{array}{lll}
x & y & z \\
z & x & y \\
y & z & x
\end{array}\right), \quad(x, y, z) \in \mathbb{C}^{3} .
$$

The mapping $A$ is linear and injective, and for all $(x, y, z),(u, v, w)$ in $\mathbb{C}^{3}$, we have

$$
A(x, y, z) A(u, v, w)=A(X, Y, Z)
$$

where

$$
\begin{aligned}
& X=u x+w y+v z \\
& Y=v x+u y+w z, \\
& Z=w x+v y+u z .
\end{aligned}
$$

Thus the condition (U) holds with $e=(1,0,0)$.

It is easy to see that condition (C 3 ) is satisfied. Indeed, consider the vectors

$$
v_{1}=(1,1,1), \quad v_{2}=\left(1, j, j^{2}\right), \quad v_{3}=\left(1, j^{2}, j\right)
$$

where $j=-\frac{1}{2}+i \frac{\sqrt{3}}{2}$ is a root of the equations $X^{3}-1=0$ and $X^{2}+X+1=0$. Then $v_{1}, v_{2}$ and $v_{3}$ are linearly independent and satisfy

$$
A(x, y, z) v_{i}=f_{i}(x, y, z) v_{i} \quad i=1,2,3,
$$

where $f_{1}, f_{2}$ and $f_{3}$ are the linear functionals

$f_{1}(x, y, z)=x+y+z, \quad f_{2}(x, y, z)=x+j y+j^{2} z, \quad f_{3}(x, y, z)=x+j^{2} y+j z$.

It is obvious that $f_{1}, f_{2}$ and $f_{3}$ are linearly independent. It follows from Theorem 4.1 that the solutions of equation $E(3)$ are given by

$$
f(x, y, z)=M_{1}(x+y+z) M_{2}\left(x+j y+j^{2} z\right) M_{3}\left(x+j^{2} y+j z\right),
$$

where $M_{1}, M_{2}$ and $M_{3}$ are multiplicative mappings from $\mathbb{C}$ to $\mathbb{C}$.

From Theorem 3.1 we deduce that the solutions of equation $E_{p}(3)$ are given by

$$
f=0, g=0, \text { and } h \text { is arbitrary }
$$


or

$$
f=0, h=0, \text { and } g \text { is arbitrary }
$$

or

$$
\begin{aligned}
& f(x, y, z)=\alpha \beta M_{1}(x+y+z) M_{2}\left(x+j y+j^{2} z\right) M_{3}\left(x+j^{2} y+j z\right), \\
& g(x, y, z)=\beta M_{1}(x+y+z) M_{2}\left(x+j y+j^{2} z\right) M_{3}\left(x+j^{2} y+j z\right), \\
& h(x, y, z)=\alpha M_{1}(x+y+z) M_{2}\left(x+j y+j^{2} z\right) M_{3}\left(x+j^{2} y+j z\right),
\end{aligned}
$$

where $M_{1}, M_{2}, M_{3}: \mathbb{C} \rightarrow \mathbb{C}$ are multiplicative mappings and $\alpha, \beta$ are arbitrary nonzero complex numbers.

It is clear that the method used in this paper may be applied to the examples listed in the introduction and to many other examples.

Acknowledgement. The authors thank very much the referee for many useful comments.

\section{References}

[1] J. Aczel and J. Dhombres, Functional Equations in Several Variables, Cambridge University Press, Cambridge, 1989.

[2] J. K. Chung and P. K. Sahoo, General solution of some functional equations related to the determinant of some symmetric matrices, Demonstratio Math. 35 (2002), No. 3, 539-544.

[3] P. K. Sahoo and T. Riedel, Mean Value Theorems and Functional Equations, World Scientific, Singapore, 1998.

DEPARTMENT OF MATHEMATICS

UNIVERSITY CADI AYYAD

FACULTY OF SCIENCES - SEMLALIA

P.O. Box: 2390

MARRAKESH, MOROCCO (MAROC)

E-mail: akkouchimo@yahoo.fr

hmlalaoui@ucam.ac.ma

Received February 12, 2004; last revised version October 21, 2004. 